\title{
Multi-modality imaging features distinguish pancreatic carcinoma from mass-forming chronic pancreatitis of the pancreatic head
}

\author{
ZHIBING RUAN $^{1}$, JUN JIAO ${ }^{1}$, DINGYU MIN ${ }^{2}$, JINHUAN QU ${ }^{1}$, JING LI ${ }^{1}$, \\ $\mathrm{JING} \mathrm{CHEN}^{1}$, QI LI ${ }^{1}$ and CHUNHONG WANG ${ }^{1}$ \\ ${ }^{1}$ Department of Radiology, Affiliated Hospital of Guizhou Medical University, Guiyang, Guizhou 550004; \\ ${ }^{2}$ Department of Medical Records and Statistics, Guizhou Provincial People's Hospital, Guiyang, Guizhou 550002, P.R. China
}

Received October 17, 2017; Accepted April 13, 2018

DOI: $10.3892 / \mathrm{ol} .2018 .8545$

\begin{abstract}
The present study retrospectively analyzed computerized tomography (CT), magnetic resonance imaging (MRI) and positron emission tomography-computerized tomography (PET/CT) data to identify features that may distinguish pancreatic carcinoma (PC) from mass-forming chronic pancreatitis (MFCP) of the pancreatic head. The mean diameter of the lesions was larger in the MFCP patients $(n=24)$ than in the PC patients $(n=30 ; 5.44 \pm 27$ vs. $3.34 \pm 1.23 \mathrm{~cm} ; \mathrm{P}<0.001)$. PC lesions showed increased lobulation when compared with the MFCP cases $(83.33$ vs. $12.5 \%$; $\mathrm{P}<0.001)$. Lesions in the MFCP patients exhibited diffuse and marginally distributed calcification. MFCP patients showed increased exudation around the lesion (83.33 vs. 13.33\%), pseudocyst formation (58.33 vs. 10\%) and thickening of the right renal fascia $(83.33$ vs. $13.33 \%$ ) than in the PC patients. MFCP patients also exhibited visible remnants of normal pancreatic tissue within the lesions. MFCP and PC patients could be distinguished by a cutoff value of $4.40 \mathrm{~cm}$ for lesion size [area under the curve (AUC): 0.894; 95\% confidence interval (CI): 0.810-0.978)], $21.85 \mathrm{Hu}$ for net-increased value in the arterial phase (AUC, 0.799; 95\% CI, 0.670-0.928), 37.70 Hu for net-increased value in the portal phase (AUC, 0.798; 95\% CI, 0.919-0.677), 4.85 for early standardized uptake value (SUV) of ${ }^{18} \mathrm{~F}$-deoxyglucose $\left({ }^{18} \mathrm{~F}-\mathrm{FDG}\right.$; AUC, 0.934; 95\% CI, 0.850-1.018) and 4.90 for delayed SUV of ${ }^{18} \mathrm{~F}-\mathrm{FDG}$ (AUC, 0.958; 95\% CI, 0.878-1.038). These findings demonstrated that the integration of data from
\end{abstract}

Correspondence to: Dr Zhibing Ruan, Department of Radiology, Affiliated Hospital of Guizhou Medical University, 28 Guiyi Street, Guiyang, Guizhou 550004, P.R. China

E-mail: zhibingruan2017@163.com

Dr Dingyu Min, Department of Medical Records and Statistics, Guizhou Provincial People's Hospital, 83 Zhongshan Road, Guiyang, Guizhou 550002, P.R. China

E-mail: mindingyu0085@163.com

Key words: pancreatic head mass, mass-forming chronic pancreatitis, pancreatic head carcinoma, multi-modality imaging, differential diagnosis dynamic contrast-enhanced CT, MRI and PET/CT imaging may distinguish MFCP from PC.

\section{Introduction}

In most cases, pancreatic carcinoma (PC) and mass-forming chronic pancreatitis (MFCP) are found in the pancreatichead, which is the largest part of the pancreas. Although these pathologies require entirely different treatment and management and have different prognoses, the overlap in their clinical and imaging features renders their early preoperative and differential diagnosis challenging (1-7). Sometimes, surgical exploratory biopsy is unable to differentiate these two pathologies. Radical surgical resection is the standard treatment for PC and the only effective means of cure. Misdiagnosis of MFCP as focal pancreatic malignancy results in unnecessary surgical treatment and misdiagnosis of PC as focal pancreatitis delays necessary surgical intervention $(1-3,8,9)$. Therefore, non-invasive imaging plays a crucial role in early differential diagnosis, personalized treatment, and evaluation of therapeutic effects in both pathologies $(3,5,7,10-20) .18^{\mathrm{F}}-\mathrm{FDG}-\mathrm{PET} / \mathrm{CT}$ is an advanced diagnostic imaging modality that combines high-resolution anatomical images from computerized tomography (CT) and functional images from positron emission tomography (PET). It is cost-effective and is used for early diagnosis, efficacy evaluation, tumor staging and prognostic evaluation and clinical treatment guidance in PC; it is particularly informative during the initial and metastasis stages of PC $(3,5,10)$. However, the specificity and sensitivity of these imaging modalities in distinguishing MFCP from PC is not known. Therefore, we retrospectively analyzed multi-modality imaging data of $30 \mathrm{PC}$ and $24 \mathrm{MFCP}$ patients to clinically distinguish PC from MFCP.

\section{Materials and methods}

Clinical data of MFCP and PC patients. The present study was approved by the Ethics Committee of the Affiliated Hospital of Guizhou Medical University (Guizhou, China) and was performed according to the Declaration of Helsinki guidelines. Written informed consent was obtained from all participants for inclusion in the study and for publishing 
their images in this manuscript. We enrolled 24 MFCP and 30 PC patients between January 2012 and May 2017 that were all confirmed by pathology [aspiration biopsy $(n=15)$ and surgical pathology $(n=9)$ in MFCP cases $(n=24)$ and surgical pathology in PC cases $(n=30)]$. All PCs were localized. The inclusion criteria were: i) all participants had been confirmed by pathology for MFCP or PC before enrollment; ii) CT (unenhanced and dynamic enhanced CT), magnetic resonance imaging (MRI) (DWI and contrast enhanced scan) and $\mathrm{PET} / \mathrm{CT}$ scans were performed within a month in all patients; and iii) general clinical data was similar for both groups. The exclusion criteria were: i) cases without pathological results; ii) incomplete data of multi-modality imaging (unenhanced and dynamic enhanced CT, MRI and PET/CT) or examination interval of all multi-modality imaging was more than a month; iii) diffused or multifocal forms of pancreatitis or diffuse nature of PC; iv) patient information did not meet the statistical requirements.

General and clinical information of the patients is shown in Table I. The 24 MFCP patients included 22 males and 2 females with a mean age of $51.92 \pm 6.7$ years. The $30 \mathrm{PC}$ patients included 19 males and 11 females with a mean age of $59.5 \pm 8.95$ years. Clinical symptoms included varying degrees of chronic intermittent or persistent pain in both MFCP and PC patients; jaundice in $18 \mathrm{MFCP}$ and 25 PC cases; weight loss of $>5 \mathrm{~kg}$ in $14 \mathrm{MFCP}$ and $23 \mathrm{PC}$ cases within 6 months; the first symptom was abdominal pain in $20 \mathrm{MFCP}$ and $21 \mathrm{PC}$ cases, whereas jaundice was the first symptom in $12 \mathrm{MFCP}$ and 19 PC cases.

Radiological examination methodology. CT scan (unenhanced and dynamic enhanced CT), MRI (DWI, MRCP, and contrast enhanced scan), and PET/CT scans were performed respectively within a month in all of patients. Besides, ERCP examination was performed in 15 cases with MFCP and 22 cases of $\mathrm{PC}$.

CT scan was obtained with a Toshiba 128-slice CT scan system. Patients were supine, and scanned from the top of the diaphragm to the lower edge of the pubic symphysis. CT scan parameters were as follows: voltage, $120 \mathrm{kV}$; current, $200 \mathrm{~mA}$; scan thickness $5 \mathrm{~mm}$, interlayer spacing $5 \mathrm{~mm}$, pitch 0.5 , and collimator 16 slice $\mathrm{x} 0.625 \mathrm{~mm}$. The original data was scanned to obtain coronal, sagittal and other orientations required for multiple planar reconstruction (MPR). The CT enhancement scan used a high-pressure injector to inject $60-100 \mathrm{ml}$ of the non-ionic iodine contrast agent iohexol (iodine $300 \mathrm{mg} / \mathrm{ml}$ ) into the cubital vein at a rate of $3 \mathrm{ml} / \mathrm{sec}$. The scan included arterial phase (delay $30 \mathrm{sec}$ ), venous phase (delay $60 \mathrm{sec}$ ) and a delay period (delay $120 \mathrm{sec}$ ). For CT non-enhanced scan, the scan was from the top of the diaphragm to the lower edge of the pubic symphysis. Patients were asked to hold their breath during scanning and breathe quietly during the scanning interval.

MRI examination was performed with a Philips Achieva 3.0-T whole-body MRI system (Philips Healthcare, Amsterdam, The Netherlands) and abdominal phased array surface coil (a 16-channel phased array coil). The patients fasted 4-8 $\mathrm{h}$ before the scan. MRI sequences are summarized in Table II. Baseline MRI included a T1-weighted turbo field-echo in-phase and opposed-phase sequence, a breath-hold multishot $\mathrm{T} 2$-weighted sequence, and a respiratory-triggered single-shot heavily T2-weighted sequence. All T2-weighted images were performed under fat saturation conditions. Dynamic images were obtained with a T1-weighted 3D turbo field-echo sequence (THRIVE). This included unenhanced phase, arterial phase (20-35 sec), portal phase (60 sec) and delayed phase $(3 \mathrm{sec})$. The time for the arterial phase imaging was determined using the MR fluoroscopic bolus detection technique. The patients were intravenously administered the contrast agent $(0.1 \mathrm{mmol} / \mathrm{kg}$ body weight gadolinium-diethylenetriaminepentaacetic acid, Gd-DTPA; Bayer Schering Pharma, Berlin, Germany) at a rate of $2 \mathrm{ml} / \mathrm{s}$ through a power injector (Mark V). Two methods of magnetic resonance cholangiopancreatography (MRCP) were used to evaluate pancreatic and bile duct anatomy. Sequence parameters of MRCP including breath-hold single-section 2D single-shot turbo spin-echo MRCP and navigator-triggered 3D turbo spin-echo MRCP were according to vendor instructions (Table II). The diffusion-weighted imaging (DWI) sequence included respiratory triggering spin echo planar imaging sequence (SE-EPI). The apparent diffusion coefficient (ADC) value was calculated with b-values of 0 and $800 \mathrm{~s} / \mathrm{mm}^{2}$. Scan data was analyzed at the Philips Achieva 3.0-T whole-body MRI system release 2.6 workstation (Philips Healthcare).

PET/CT examination was performed in a Philips GEMINI TF 64 PET/CT machine (Philips Healthcare) with ${ }^{18} \mathrm{~F}$-deoxyglucose (FDG; purity $>95 \%$ ). Patients fasted for $6 \mathrm{~h}$ before the scan, and the fingertip fasting blood glucose level was $<11 \mathrm{mmol} / 1$ (normal range: 3.9-6.1 mmol/l). We injected ${ }^{18} \mathrm{~F}-\mathrm{FDG}$ (3.7 MBq/kg body weight) into the cubital vein and the examination was performed after supine rest for $\sim 1 \mathrm{~h}$ after the injection in a quiet dark room. CT scan parameters were as follows: voltage $120 \mathrm{kV}$, current $120 \mathrm{~mA}$, CT reconstruction thickness $5 \mathrm{~mm}$ and an interval of $5.0 \mathrm{~mm}$. The PET images were attenuated and corrected with CT data and ordered subset iterative expectation maximization (OSEM) for PET image reconstruction was performed with a thickness of $5 \mathrm{~mm}$ and interval distance of $5 \mathrm{~mm}$. Reconstruction images of CT and PET were transferred to a Philips dedicated workstation to obtain cross-sectional PET, sagittal PET, coronal PET, CT and PET/CT fusion images. Abnormal lesions were outlined as the region of interest (ROI) using a semi-quantitative method to measure the maximum standardized uptake value (SUVmax) of radioactivity.

Analysis of multi-modality imaging data. The images were reviewed independently by two expert radiologists and a consensus diagnosis was arrived at. The following key indicators were recorded at the Philips workstation: i) pancreatic tumor size, shape, density or characteristic signals such as multi-phase enhanced characteristics and the boundary; ii presence or absence of cystic lesions of tumor and surrounding area; iii) style and degree of dilation of the main pancreatic duct and common bile duct; more attention was paid to common bile duct lesions with or without interruption and stenosis, with or without mural nodules, and with or without duct penetration (mass segment with pancreatic duct stenosis without wall irregularities, or mass without stenosis) and 'double' or bile duct 'disjoint' signs (segments of the common bile duct and the pancreatic duct are cut off due to pancreatic cancer invasion; 
Table I. General information of the enrolled MFCP and PC patients.

\begin{tabular}{|c|c|c|c|c|}
\hline Parameter & $\operatorname{MFCP}(n=24)$ & $\mathrm{PC}(\mathrm{n}=30)$ & $\chi^{2}$ or t-value & P-value \\
\hline Males $[\mathrm{n}(\%)]$ & $22(91.67)$ & $19(63.33)$ & 5.856 & 0.016 \\
\hline Age, years (mean \pm SD) & $51.92 \pm 6.7$ & $59.5 \pm 8.95$ & $-1.632^{\mathrm{t}}$ & 0.109 \\
\hline History of long-term drinking [n (\%)] & $16(66.67)$ & $13(43.33)$ & 2.920 & 0.088 \\
\hline History of biliary tract disease [n (\%)] & $20(83.33)$ & $5(16.67)$ & 23.834 & $<0.001$ \\
\hline History of pancreatitis [n (\%)] & $18(75 \%)^{\mathrm{a}}$ & $3(10)$ & 23.704 & $<0.001$ \\
\hline
\end{tabular}

${ }^{\mathrm{a}} \geq 2$ episodes in 6 cases; $\mathrm{t}$, independent-samples t-test; SD, standard deviation; MFCP, mass-forming chronic pancreatitis; PC, pancreatic carcinoma.

Table II. Magnetic resonance imaging sequences and parameters.

\begin{tabular}{|c|c|c|c|c|c|c|c|c|c|}
\hline $\begin{array}{l}\text { MRI } \\
\text { sequence }\end{array}$ & $\begin{array}{l}\mathrm{TR} / \mathrm{TE} \\
\text { (msec) }\end{array}$ & $\begin{array}{l}\text { Flip } \\
\text { angle } \\
\left(^{\circ}\right)\end{array}$ & $\begin{array}{c}\text { Slice } \\
\text { thickness } \\
(\mathrm{mm})\end{array}$ & $\begin{array}{l}\text { Slice } \\
\text { gap } \\
(\mathrm{mm})\end{array}$ & $\begin{array}{l}\text { Matrix } \\
\text { size }\end{array}$ & $\begin{array}{c}\text { Band width } \\
\text { (Hz/pixel) }\end{array}$ & $\begin{array}{l}\text { FOV } \\
(\mathrm{cm})\end{array}$ & $\begin{array}{c}\text { Acquisition } \\
\text { time (sec) }\end{array}$ & $\begin{array}{c}\text { No. of } \\
\text { excitations }\end{array}$ \\
\hline T1W 2D dual GRE & $5 / 1.14-2.3$ & 15 & 6 & 1 & $252 \times 159$ & 434 & 37.5 & 14.4 & 1 \\
\hline BH-MS-T2WI & $1,410 / 80$ & 90 & 7 & 1 & $320 \times 224$ & 641.5 & 37.5 & 46.2 & 1 \\
\hline RT-SS-T2WI & $1,277 / 70$ & 90 & 7 & 1 & $236 \times 166$ & 549.3 & 37.5 & - & 2 \\
\hline RT-SS-HT2WI & $2,580 / 740$ & 90 & 5 & 1 & $256 \times 153$ & 591.9 & 37.5 & - & 1 \\
\hline RT-3D-GRE T1WI & $3.0 / 1.39$ & 10 & 2 & 1 & $252 \times 197$ & 723.4 & 37.5 & 15 & 2 \\
\hline BH-2D-MRCP & $4,800 / 894$ & 90 & 40 & 1 & $256 \times 192$ & 408 & 30 & 9 & 1 \\
\hline NT-3D-MRCP & $1,673 / 740$ & 90 & 1 & 1 & $256 \times 153$ & 591.9 & 36 & 250 & 1 \\
\hline DWI & $1,600 / 70$ & 90 & 7 & 1 & $124 \times 100$ & 43.9 & 36 & 174 & 4 \\
\hline
\end{tabular}

MRI, magnetic resonance imaging; DWI, diffusion-weighted imaging; MRCP, magnetic resonance cholangiopancreatography; TR, repetition time; TE, echo time; 3D, three-dimensional; GRE, gradient recalled echo; BH-MS-T2WI, Breath-hold multishot T2-weighted; RT-SS-T2WI, Respiratory-triggered single-shot T2-weighted imaging; RT-SS-HT2WI, Respiratory-triggered single-shot heavily T2-weighted imaging; RT-3D-GRE T1WI, T1-weighted 3D gradient-recalled echo; BH-2D-MRCP, Breath-hold 2D MRCP; NT-3D-MRCP, Navigator-triggered 3D MRCP; dash (-) indicates variable value depending on patient breathing and number of respiratory interval in respiratory-triggered techniques; FOV, field of view.

the common bile duct and the pancreatic duct expand, but do not extend into the tumor); iv) whether peripancreatic vascular tissue was violated (the fat disappears between the mass and adjacent vessels, and the mass wraps around the adjacent vessel at $>180^{\circ}$ ) and the presence or absence of blocked blood vessels and presence of stenosis; and v) adjacent tissue and organ changes, including the presence or absence of abdominal and retroperitoneal lymph node metastasis, liver and spleen metastasis, or renal fascia thickening. We also collected and recorded clinical data, including symptoms, laboratory tests and pathology reports after surgery. The image analysis and diagnosis of each patient were compared with the pathology results.

Statistical analysis. SPSS 19.0 statistical software (IBM Corp., Armonk, NY, USA) was used to analyze the data. For data with normal distribution with homogeneity of variance test, data were presented as the means \pm standard deviation (mean $\pm \mathrm{SD}$ ). We used the independent-samples t-test and $\chi^{2}$ comparison test or Fisher's exact test. In ROC analysis, the appropriate cutoff value of lesion size, net-increased value in arterial and portal phases as well as early and delayed SUV values corresponding to the maximal Youden index was determined. We also determined the sensitivity and specificity values of significant imaging findings. The significance level was set at $\mathrm{P}<0.05$ (two-tailed).

\section{Results}

Distinguishing multi-modality imaging features in $P C$ and $M F C P$. The lesion volume was higher in the MFCP group than in pancreatic cancer $(5.44 \pm 1.27 \mathrm{~cm}$ vs. $3.34 \pm 1.23 \mathrm{~cm}$; $\mathrm{P}<0.001$; Figs. 1-4; Table III). The right perirenal fascia thickening was observed in $83.33 \%$ of MFCP and $13.33 \% \mathrm{PC}$ patients $(\mathrm{P}<0.001$; Figs. $1 \mathrm{~A}$ and $\mathrm{B}, 2 \mathrm{~A}$ and $\mathrm{B}, 3 \mathrm{~A}-\mathrm{C}, 4 \mathrm{~A}$ and $\mathrm{B}$, $5 \mathrm{~A}, \mathrm{~B}$ and $\mathrm{D}$ and 6A-D). Lobulation was observed in $83.33 \%$ of PC and $12.5 \%$ of MFCP patients $(\mathrm{P}<0.001$; Table III; Figs. 1A-6A).

Cystic necrosis was observed in $60 \%$ PC and $29.17 \%$ MFCP patients $(\mathrm{P}<0.05$; Table III). Calcification was observed in $58.33 \%$ MFCP and $10 \%$ PC patients $(\mathrm{P}<0.001$; Table III). Predominantly, calcification in MFCP patients was mixed type $(8 / 14 ; 57.14 \%)$, characterized by diffuse pattern and marginal distribution of the lesions (Figs. 1A and 2B). Furthermore, 

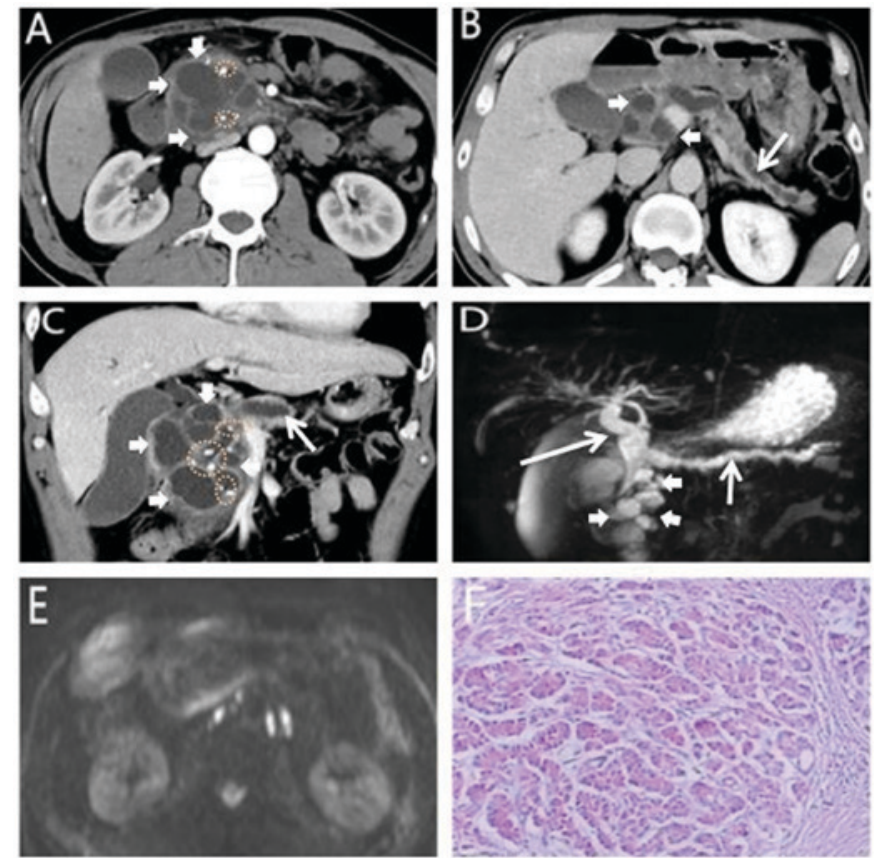

Figure 1. A 49-year-old male MFCP patient with epigastric pain for 6 months The pancreatic head (indicated by arrows) exhibited multiple honeycombs (indicated by arrowhead) of mass with (A) homogeneous enhancement in the enhanced CT arterial phase and (B) in the enhanced CT venous phase as well as (C) in the coronal reconstruction. This included slight enhancement of the cystic wall and the intracystic component without enhancement. The cystic lesions presented tension and multiple calcifications (annulus) and was accompanied by exudation around the pancreas, expansion of the distal main pancreatic duct expansion (indicated by arrows) and atrophy of the pancreatic body and tail. (D) The image shows multiple cystic lesions (indicated by arrowheads) with clear tension and expansion of bile duct and pancreatic duct (indicated by arrows) and the dilated bile duct and pancreatic duct traversing through the lesion area. (E) Diffusion-weighted imaging showed the pancreatic head mass with unrestricted diffusion. (F) Hematoxylin and eosin stained cross section (magnification, $\mathrm{x} 100$ ) revealed chronic inflammation of the pancreas with a dilated duct, focal fibrosis and pancreatic tissue necrosis MFCP, mass-forming chronic pancreatitis; CT, computerized tomography.

visible remnants of normal pancreatic tissue were observed in the MFCP lesions (Figs. 1A, 2B and 3B).

Pseudocyst formation was observed in $58.33 \%$ MFCP and $10 \%$ PC patients $(\mathrm{P}<0.001$; Table III). Honeycomb-like cystic lesions were found only in the MFCP group (Figs. 1C and D, 2B and 3B). Atrophy of the pancreatic body and tail was observed in $41.67 \% \mathrm{MFCP}$ and $36.67 \% \mathrm{PC}$ patients ( $\mathrm{P}=0.708$; Figs. 1B, 2A and 3C). Dilated bile ducts passed through the lesion areas in 79.17\% MFCP and $16.67 \%$ PC cases (P<0.001; Table III; Figs. 1D, 2C and 4B). Dilated pancreatic ducts interrupted the lesion areas in $8.33 \% \mathrm{MFCP}$ and $70 \%$ PC cases $(\mathrm{P}<0.001$; Table III; Fig. 5C). However, the double duct sign, which indicates the expansion of both the pancreatic duct and the bile duct, was similar in both groups $(\mathrm{P}=0.20$; Table III). The net increase in arterial and portal venous phase enhancements was higher in the MFCP group than in the $\mathrm{PC}$ group $(\mathrm{P}<0.001$ and $\mathrm{P}=0.008$, respectively; Table III). Peripancreatic vascular invasion was similar in both groups (Table III). However, we observed 7 cases of cancer embolus and 5 cases of hepatic metastases in PC, thereby showing increased vascular invasion in PC. Meanwhile, 2 MFCP patients showed portal vein thrombosis (Fig. 4B),
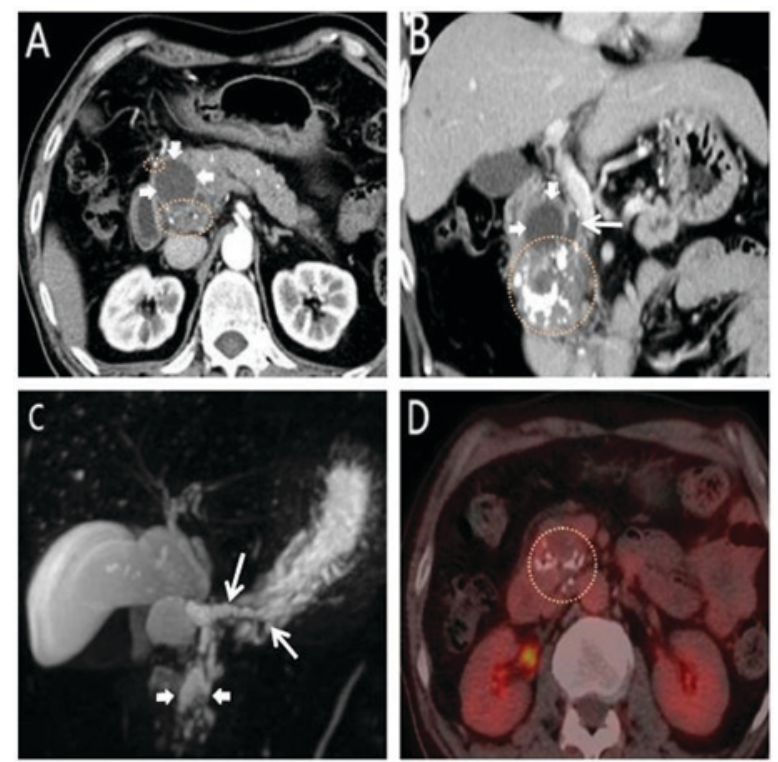

Figure 2. A 56-year-old male MFCP patient with upper abdominal pain and discomfort for 10 days. CT image exhibited enlarged and misshaped pancreatic head with enhancement of uneven decrease in the $(\mathrm{A})$ enhanced CT arterial phase and (B) enhanced CT venous phase in the coronal reconstruction. The multiple cystic lesions (indicated by arrowheads) were not enhanced but with tension, and the inner wall was light and finished, combined with multiple calcified nodules that were distributed at the edges and were patchy (annulus). The dilated common bile duct and pancreatic duct (indicated by arrows) traverse through the lesion area. (C) The image clearly reveals the bile and the pancreatic ducts (indicated by arrow). (D) The ${ }^{18} \mathrm{~F}$-deoxyglucose-positron emission tomography/CT scan image showed normal metabolism in the mass of the pancreatic head. MFCP, mass-forming chronic pancreatitis; CT, computerized tomography.
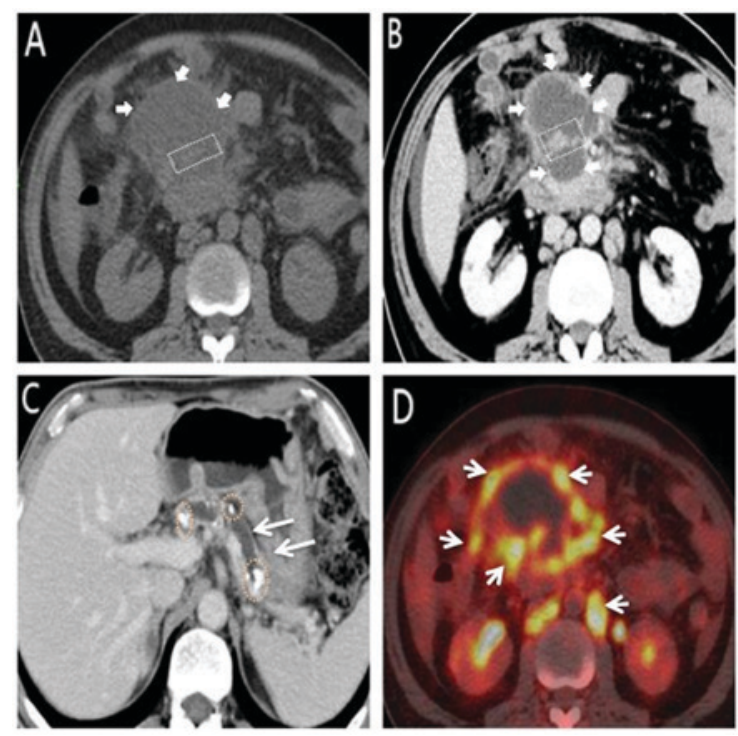

Figure 3. A 41-year-old male MFCP patient with recurrent abdominal pain and fever for 3 months. CT scan images revealed the enlarged and misshaped head of the pancreas, with (A) enhancement of uneven decrease at enhanced CT arterial phase and ( $\mathrm{B}$ and $\mathrm{C}$ ) enhanced $\mathrm{CT}$ venous phase. The rectangle in images $\mathrm{A}$ and $\mathrm{B}$ shows the normal pancreatic tissue in the lesion. The multilocular cystic lesions show no enhancement with tension, however, they have slight enhancement of the inner wall, accompanied with pancreatic duct dilatation (indicated by arrow), multiple stones and calcifications (annulus) in the atrophied pancreatic body and tail. (D) ${ }^{18} \mathrm{~F}$-deoxyglucose-positron emission tomography/CT scan image shows that the metabolism of the diffused cystic areas of mass lesion was not increased. There was also significantly high uptake around the lesion and many adjacent lymph nodes (indicated by short arrows). MFCP, mass-forming chronic pancreatitis; CT, computerized tomography. 
Table III. Comparison of multi-modality imaging features between MFCP and PC cases of the pancreatic head.

\begin{tabular}{|c|c|c|c|c|}
\hline Parameter & $\operatorname{MFCP}(n=24)$ & $\mathrm{PC}(\mathrm{n}=30)$ & $\chi^{2}$ or t-value & P-value \\
\hline Lesion size $(\mathrm{cm}$; mean $\pm \mathrm{SD})$ & $5.44 \pm 1.27$ & $3.34 \pm 1.23$ & $6.140^{\mathrm{t}}$ & $<0.001$ \\
\hline Lobulation [n (\%)] & $3(12.5)$ & $25(83.33)$ & 26.796 & $<0.001$ \\
\hline Calcification [n (\%)] & $14(58.33)$ & $3(10)$ & 14.440 & $<0.001$ \\
\hline Patchy & $4(28.57)$ & $0(0)$ & - & - \\
\hline Dot & $2(14.29)$ & $2(66.67)$ & - & - \\
\hline Hybrid & $8(57.14)$ & $1(33.33)$ & 8.640 & 0.007 \\
\hline Cystic necrosis [n (\%)] & $7(29.17)$ & $18(60)$ & 5.098 & 0.024 \\
\hline \multicolumn{5}{|l|}{ Net-increased value of CT enhancement $(\mathrm{Hu}$, mean $\pm \mathrm{SD})$} \\
\hline Arterial phase & $24.42 \pm 9.81$ & $13.92 \pm 5.50$ & 4.974 & $<0.001$ \\
\hline Pancreatic parenchymal phase (portal phase) & $45.41 \pm 14.56$ & $35.83 \pm 10.19$ & 2.840 & 0.008 \\
\hline High signal on DWI [n (\%)] & $2(28.57)$ & $17(89.47)$ & 13.658 & $<0.001$ \\
\hline ADC value $(\text { mean } \pm S D)^{a}$ & $1.36 \pm 0.13$ & $1.01 \pm 0.11$ & 10.703 & $<0.001$ \\
\hline Early SUV value (mean \pm SD ) & $2.51 \pm 0.42$ & $7.13 \pm 3.04$ & -7.375 & $<0.001$ \\
\hline Delayed SUV value (mean \pm SD ) & $2.10 \pm 0.31$ & $9.20 \pm 3.56$ & -9.723 & $<0.001$ \\
\hline Atrophy of the pancreatic body and tail [n (\%)] & $10(41.67)$ & $11(36.67)$ & -0.140 & 0.708 \\
\hline Pseudocyst $[\mathrm{n}(\%)]$ & $14(58.33)$ & $3(10)$ & 14.440 & $<0.001$ \\
\hline Pancreatic duct expansion [n (\%)] & $16(66.67)$ & $20(66.67)$ & 0.000 & 0.995 \\
\hline Uniformity of expansion & $2(12.5)$ & $18(90)$ & 15.263 & $<0.001$ \\
\hline Beaded expansion & $14(87.5)$ & $2(10)$ & 17.070 & $<0.001$ \\
\hline Pancreatolithiasis & $13(54.17)$ & $5(16.67)$ & 8.438 & 0.004 \\
\hline Bile duct penetration sign [n (\%)] & $19(79.17)$ & $5(16.67)$ & 21.094 & $<0.001$ \\
\hline Cholangiectasis [n (\%)] & $17(70.83)$ & $16(53.33)$ & 1.720 & 0.190 \\
\hline Double duct sign [n (\%)] & $7(29.17)$ & $14(46.67)$ & 1.713 & 0.200 \\
\hline Disjoint sign of the bile duct and pancreatic duct [n (\%)] & $2(8.33)$ & $21(70)$ & 20.737 & $<0.001$ \\
\hline Right anterior renal fascia thickening [n (\%)] & $20(83.33)$ & $4(13.33)$ & 26.460 & $<0.001$ \\
\hline Peripancreatic vascular invasion $[\mathrm{n}(\%)]^{\mathrm{b}}$ & $17(70.83)$ & $19(63.33)$ & 0.337 & 0.561 \\
\hline Retroperitoneal lymph nodes enlargement $[\mathrm{n}(\%)]^{\mathrm{c}}$ & $11(45.83)$ & $21(70)$ & 3.225 & 0.073 \\
\hline
\end{tabular}

${ }^{\mathrm{a}} \mathrm{ADC}$ values based on DWI scan were statistically significant in 7 cases of MFCP and 19 cases of PC $\left[(1.43 \pm 0.20) \times 10^{-3} \mathrm{~mm}{ }^{2} ; \mathrm{P}<0.05\right]$; ${ }^{b} \mathrm{PC}$ group showed increased tumor vessel invasion and abnormal morphology, while the majority of MFCP cases showed peripancreatic vascular

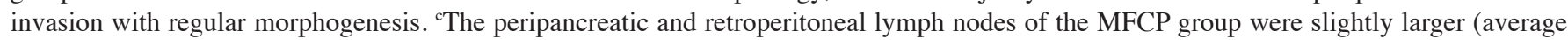
diameter, $\sim 11 \mathrm{~mm}$ ), with edge blur and mainly mild homogeneous enhancement. The PC group showed significantly larger lymph nodes (average diameter, $\sim 15 \mathrm{~mm}$ ) with significantly enhanced base or ring enhancement; t, independent-samples t-test; Dash (-) indicates that statistical analysis was not performed; SD, standard deviation; MFCP, mass-forming chronic pancreatitis; PC, pancreatic carcinoma; SUV, standardized uptake values; CT, computerized tomography.

suggesting initiation of peripancreatic vascular invasion in MFCP by regular morphogenesis.

We observed that there were no significant differences in pancreatic and retroperitoneal lymph nodes of both MFCP and PC groups. In MFCP, the multiple lymph nodes were slightly larger than normal (average diameter: $11 \mathrm{~mm}$ vs. $<5 \mathrm{~mm}$ ) with mild homogeneous enhancement, whereas, the PC group showed a significant increase (average diameter: $15 \mathrm{~mm}$ ) with partially visible fusion and uniform annular enhancement.

High signal on DWI was observed in $28.57 \%$ MFCP and 89.47\% PC cases $(\mathrm{P}<0.001$; Table III; Fig. 1E). Moreover, $\mathrm{ADC}$ values were higher in the MFCP than in the PC group $(\mathrm{P}<0.001$; Table III). The MFCP group showed lower maximal mean SUV than in the PC group for the early $(2.51 \pm 0.42 \mathrm{vs}$.

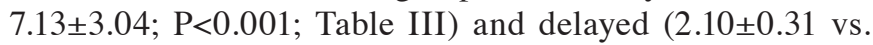

9.20 $3.56 ; \mathrm{P}<0.001$; Table III) phases. The delayed phase SUV increased in the PC group (Figs. 5E and 6G and $\mathrm{H}$ ), but changed minimally or decreased in the MFCP group (Figs. 2D and 4C and D).

As shown in Table IV and Figs. 7 and 8, the optimal cutoff values for distinguishing MFCP from PC patients was $4.40 \mathrm{~cm}$ for lesion size (sensitivity, 75\%; specificity, 90\%; area under the curve (AUC): $0.894 ; 95 \%$ confidence interval (CI): $0.810-0.978), 21.85 \mathrm{Hu}$ for net-increased value in arterial phase (sensitivity, 58\%; specificity, 97\%; AUC, 0.799; 95\% CI, 0.670-0.928), 37.70 Hu for net-increased value in portal phase (sensitivity, 83\%; specificity, 70\%; AUC, 0.798; 95\% CI, 0.919-0.677), 4.85 for early SUV value (sensitivity, 90\%; specificity, 92\%; AUC, 0.934; 95\% CI, 0.850-1.018) and 4.90 for delayed SUV value (sensitivity, $97 \%$; specificity, $96 \%$; AUC, 0.958; 95\% CI, 0.878-1.038). 
Table IV. Diagnostic performance of significant imaging features in distinguishing MFCP from PC.

\begin{tabular}{lcccccc}
\hline Parameter & AUC & $95 \% \mathrm{CI}$ & P-value & Cut off point & Sensitivity & Specificity \\
\hline Lesion size $(\mathrm{cm})$ & 0.894 & $0.810-0.978$ & $<0.001$ & $4.40 \mathrm{~cm}$ & 0.75 & 0.90 \\
Net-increased value in arterial phase $(\mathrm{Hu})$ & 0.799 & $0.670-0.928$ & $<0.001$ & $21.85 \mathrm{Hu}$ & 0.58 & 0.97 \\
Net-increased value in portal phase $(\mathrm{Hu})$ & 0.798 & $0.919-0.677$ & $<0.001$ & $37.70 \mathrm{Hu}$ & 0.83 & 0.70 \\
Early SUV & 0.934 & $0.850-1.018$ & $<0.001$ & 4.85 & 0.90 & 0.92 \\
Delayed SUV & 0.958 & $0.878-1.038$ & $<0.001$ & 4.90 & 0.97 & 0.96 \\
\hline
\end{tabular}

MFCP, mass-forming chronic pancreatitis; PC, pancreatic carcinoma; AUC, area under the curve; CI, confidence interval; Hu, Hounsfield unit; SUV, standardized uptake values.
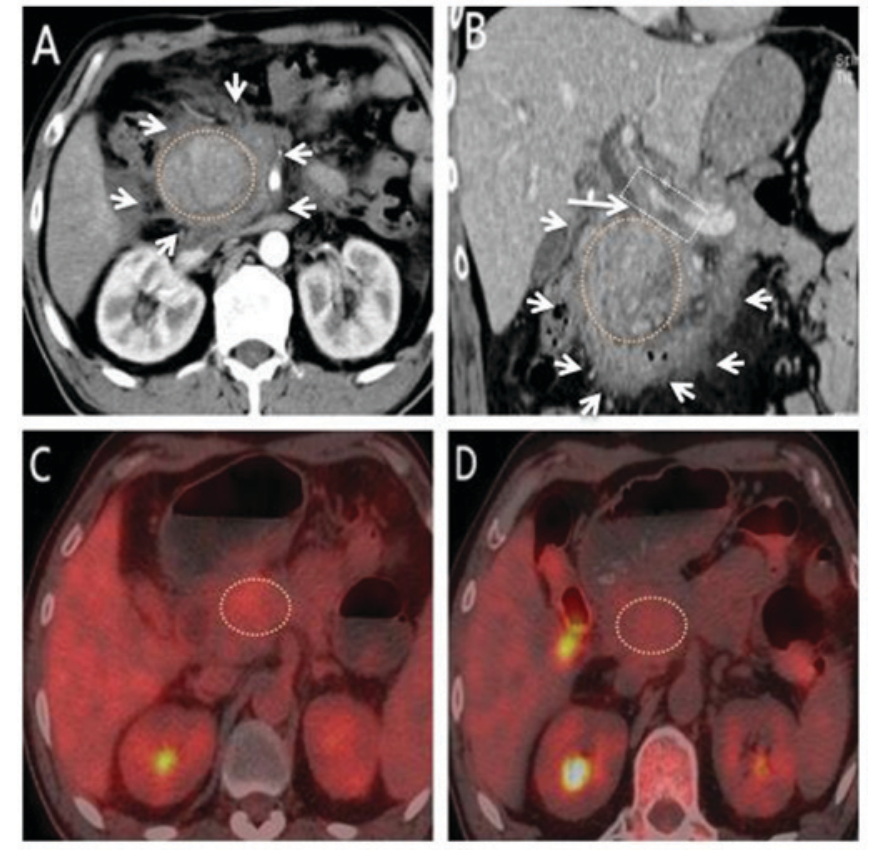

Figure 4. A 59-year-old male MFCP patient with upper abdominal pain for 12 days and a 7-year history of necrotizing pancreatitis. CT scan image revealed a huge mass of the pancreatic head that is slightly uneven and showed (A) progressive enhancement (indicated by dotted circle) in the enhanced CT arterial phase and (B) enhanced CT venous phase reconstruction. There was significant peripancreatic exudation (indicated by short arrows) and perirenal fascia thickening. The dilated pancreatic duct and the common bile duct show 'rat-tailed' stenosis (indicated by arrows) within and through the lesion area, and was associated with portal vein thrombosis (indicated by a rectangle). ${ }^{18} \mathrm{~F}$-deoxyglucose-positron emission tomography/CT image revealed (C) higher uptake at the early phase and (D) decreased uptake at the delayed phase in the pancreatic head mass (indicated by a dotted circle). MFCP, mass-forming chronic pancreatitis; CT, computerized tomography.

\section{Discussion}

Pancreatic cancer is a common and aggressive abdominal malignant tumor with a 5-year survival rate of less than $5 \%$ in patients that undergo surgical resection; $50-70 \%$ of cases occur in the head of the pancreas $(3,8)$. MFCP accounts for $15-30 \%$ of chronic pancreatitis, wherein protracted pancreatic inflammation results in the fibrotic replacement of the destroyed pancreatic parenchyma and formation of a local mass due to chronic inflammatory cell infiltration $(3,4,21,22)$. MFCP and PC are similar in various clinical and biological aspects, and chronic pancreatitis is one of the risk factors for
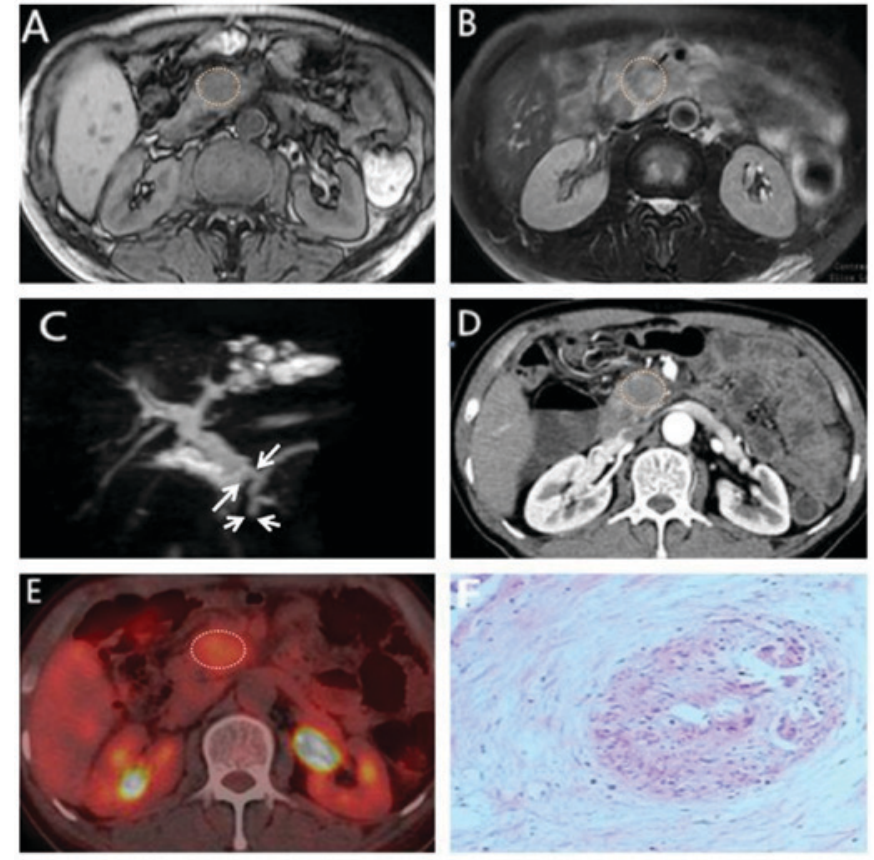

Figure 5. A 56-year-old male PC patient with upper abdominal pain for 2 months with a change in bowel habits for 1 month. CT scan image showed enlarged and deformed pancreatic head with a nodule, which revealed a (A) lower uneven signal at the T1WI in-phase and (B) a higher uneven signal at T2WI fat-saturation in the pancreatic head (indicated by a dotted circle). (C) The common bile duct (indicated by arrows) and pancreatic duct (indicated by short arrow) are 'disjoint' and show uniform dilatation. (D) Pancreatic head nodule (indicated by dotted circle) showed uneven lower signal in the enhanced CT arterial phase with an unclear boundary. (E) Positron emission tomography/CT image revealed higher uptake in the pancreatic head nodule (indicated by a dotted circle). (F) Hematoxylin and eosin stained section shows moderately differentiated adenocarcinoma in the pancreatic head (magnification, x100). PC, pancreatic carcinoma; CT, computerized tomography.

pancreatic cancer $(2,4,7)$. In our study, MFCP patients were younger than PC patients, though statistically insignificant. The MFCP patients, unlike PC patients, had a history of biliary tract disease, long-term alcohol consumption and chronic pancreatitis. This suggests that chronic damage because of biliary disease, long-term alcohol drinking and a history of pancreatitis are independent risk factors for MFCP (22). In our study, we distinguished MFCP and PC by analyzing multiple Multi-modality imaging features.

The size of the lesions was larger in MFCP patients than in PC patients and an optimal cutoff value of 4.40 distinguished 

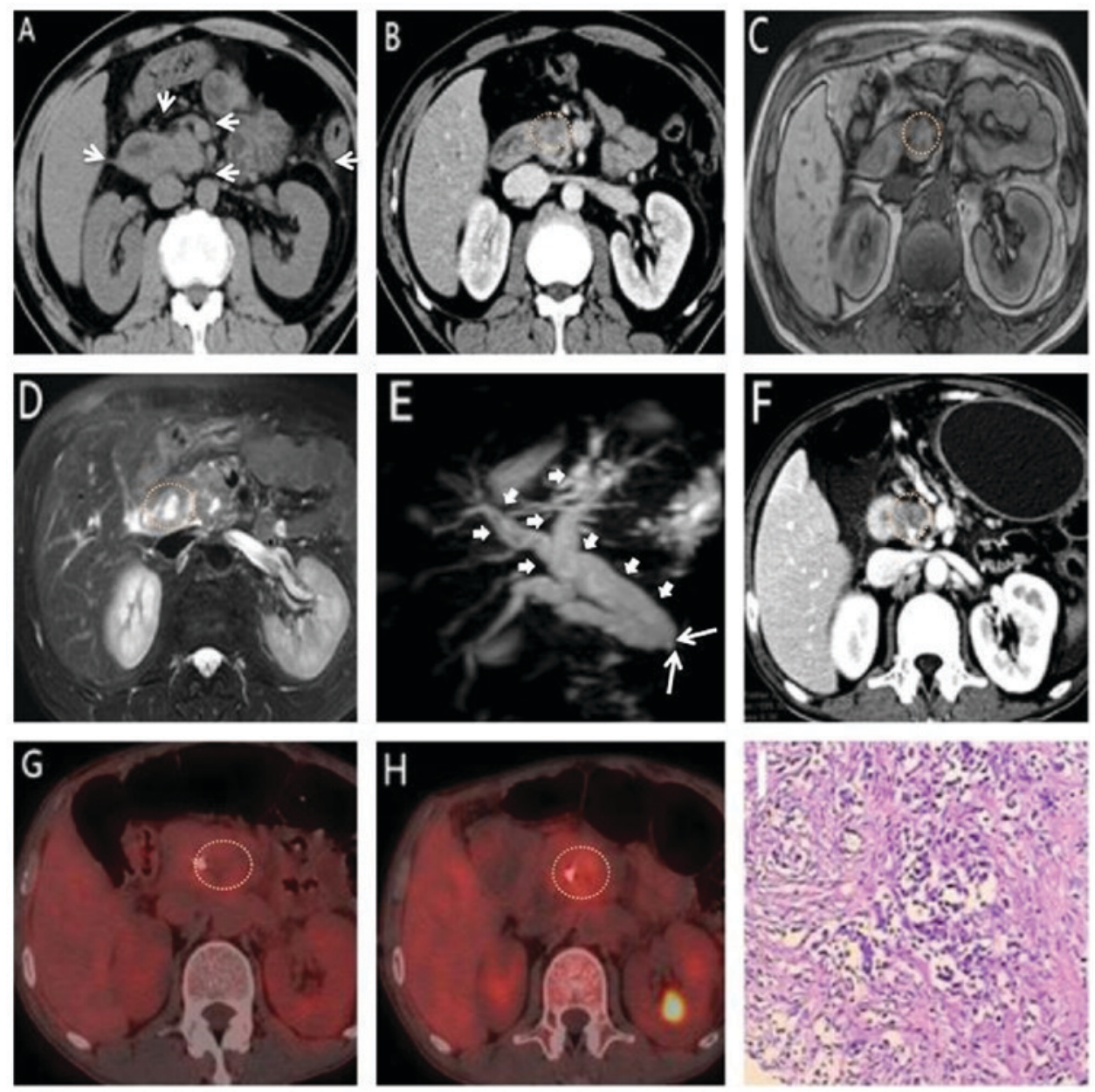

Figure 6. A 57-year-old male PC patient with abdominal pain and jaundice 9 months following cholecystectomy. (A) CT scan image showed pancreatic head without significant abnormal nodule or mass in the plain CT axial scan with significant exudation (indicated by short arrows) around the pancreas and (B) a nodule (indicated by a dotted circle) with uneven lower enhancement in the enhanced CT arterial phase. (C) The pancreatic head nodule (indicated by a dotted circle) showed lower signal at T1WI and (D) higher uneven signal at T2WI. (E) Magnetic resonance cholangiopancreatography revealed the common bile duct (indicated by arrows) and the pancreatic duct in the pancreatic lesion area. The uniform distal duct expansion (indicated by arrowheads) is also seen. (F) Re-examination following 8 months revealed enlarged pancreatic nodule with increased exudate around the lesion (indicated by a dotted circle) than before in the enhanced CT. (G) Positron emission tomography/CT image shows higher uptake at the early phase and (H) higher uptake at delayed phase in the pancreatic head nodule (indicated by dotted circles). (I) Hematoxylin and eosin stained image reveals moderately differentiated adenocarcinoma of the pancreatic nodule (magnification, $\mathrm{x} 400$ ). PC, pancreatic carcinoma; CT, computerized tomography.

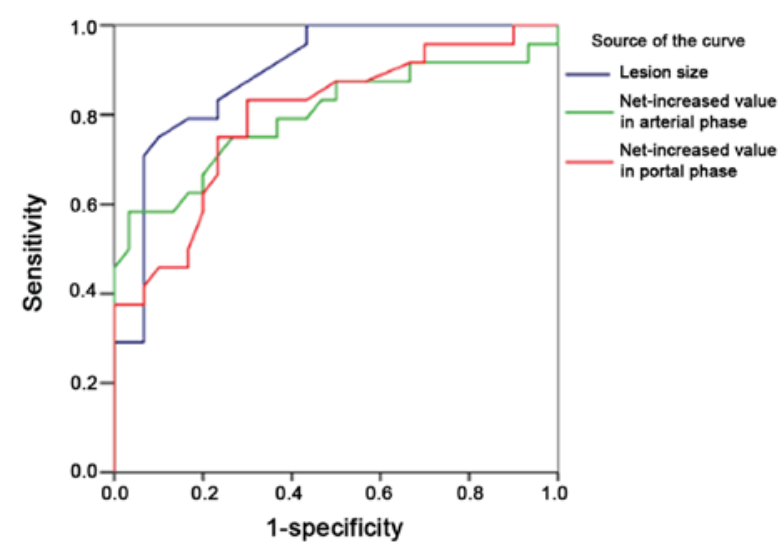

Figure 7. Receiver operating characteristic curves for lesion size and net-increased standardized uptake values in arterial and portal phases to distinguish MFCP and PC. The cutoff value was 4.40 (sensitivity, 75\%; specificity, $90 \%$; AUC, $0.894 ; 95 \%$ CI, $0.810-0.978$ ) for lesion size; 21.85 (sensitivity, 58\%; specificity, 97\%; AUC, 0.799; 95\% CI, 0.670-0.928) for arterial phase and 37.70 (sensitivity, $83 \%$; specificity, $70 \%$; AUC, 0.798 ; 95\% CI, 0.919-0.677) for portal phase. CI, confidence interval; AUC, area under the curve.

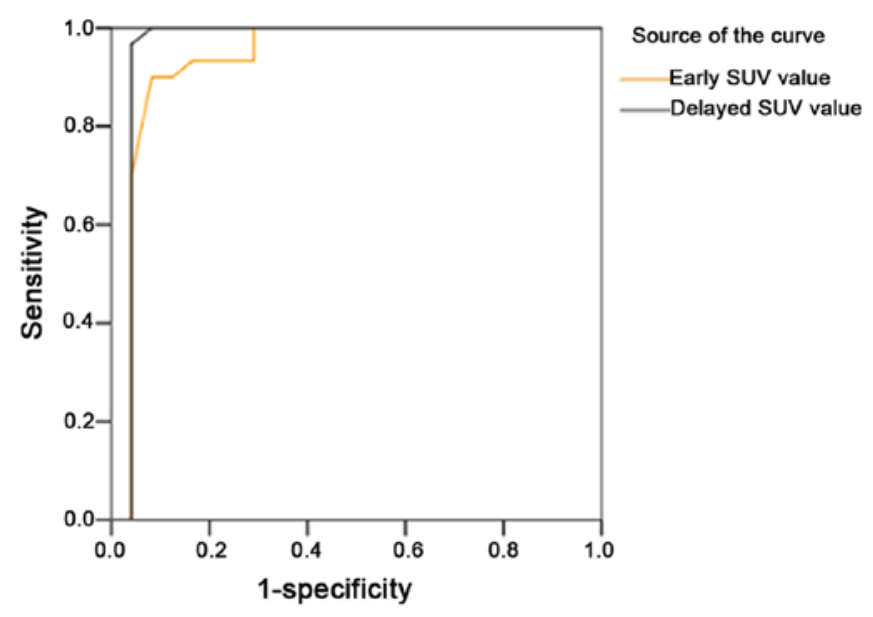

Figure 8. Receiver operating characteristic curve for early and delayed SUV to distinguish MFCP and PC. The cutoff value is 4.85 (sensitivity, $90 \%$; specificity, 92\%; AUC, 0.934; 95\% CI, 0.850-1.018) for early SUV values and 4.90 (sensitivity, 97\%; specificity, 96\%; AUC, 0.958; 95\% CI, 0.878-1.038) for delayed SUV. CI, confidence interval; AUC, area under the curve; SUV, standardized uptake values. 
MFCP from PC lesions. The boundaries of the lesions were more unclear in MFCP than in pancreatic cancer, and the exudation around the lesion was more obvious in the MFCP group. The right perirenal fascia thickening was more obvious in MFCP than in PC, probably due to chronic inflammation. Moreover, the lack of a complete pancreatic capsule resulted in inflammatory exudate, connective tissue proliferation and necrosis, which easily spread into the adjacent peripancreatic and renal interstitial spaces. Anterior renal fascia or renal peritoneal thickening was strong evidence of chronic pancreatic inflammation. Moreover, lobulation was closely related to lesion size, with predominantly larger lesions observed in PC cases than in MFCP (83.33\% vs. $12.5 \%)$. These parameters highlighted the distinct biological and pathophysiological properties of PC and MFCP.

Cystic necrosis was more common in PC than in the MFCP group. In PC, the wall of cystic necrosis was uneven, whereas the inner margin of the MFCP cystic necrosis area was smoother. Calcification was more common in MFCP than in PC. The main calcification type was mixed with diffused distribution. The edge of the lesion was associated with multiple pancreatic duct stones because of recurrent and progressive inflammatory fibrosis that damaged the internal and external secretory functions to varying degrees and resulted in calcium deposition. These, subsequently lead to pancreatic duct calcification and stones. In PC, calcification was located in the central necrotic area. These common changes occurred in both diseases. However, we observed visible remnants of normal pancreatic tissue within MFCP lesions that could be used to distinguish MFCP from PC. Large scale studies are necessary to confirm these differences.

Cystic lesions or pancreatic pseudocysts are fluid-filled cavities wrapped by the wall of fibrotic pancreatic tissue. The incidence of cystic lesions was observed in $58.33 \%$ of MFCP patients and $10 \%$ of PC patients. In MFCP, cystic lesions were mainly located within or around the pancreatic head mass and characterized by honeycomb-like cysts as well as calcified and discontinuous wall. These features were not observed in the PC cases.

In MFCP, secondary chronic pancreatitis causes atrophy of the pancreatic body and tail. Atrophy and pancreatic duct obstruction of the body and tail of the pancreas have been linked to PC (3). In our study, the incidence of atrophy was similar between MFCP and PC cases (41.67\% vs. 36.67\%). A previous report indicated differences in atrophy at an early stage of PC (3), which needs to be confirmed by large scale studies in the future.

In most PC cases, the pancreatic duct was obstructed by infiltrative growth of the cancer resulting in distal pancreatic duct dilation, which was smooth and continuous. However, few PC cases showed no expansion of the distal pancreatic duct as compensation to obstruction. Therefore, mild expansion of the pancreatic duct is an early indicator of PC. Furthermore, obstruction of the common bile duct was observed in most PC cases. The dilation of the pancreatic duct in MFCP showed beaded appearance and was secondary to the chronic pancreatitis. It showed pancreatic duct stones and calcification and compressed the distal common bile duct. Infiltration of the bile wall by inflammatory cells and fibrosis resulted in incomplete biliary obstruction. A previous study showed that bile duct dilation was similar in both MFCP and PC, but the degree of dilatation was more obvious in $\mathrm{PC}$, probably related to the pathological stages of the samples and other molecular mechanisms (23-26). Furthermore, the common bile duct wall showed a reinforced ring at the delayed phase in MFCP samples. Further analysis is necessary to confirm if this is a distinguishing feature that can be used to distinguish MFCP from PC. The dilated pancreatic ducts passed through the lesion area in $79.17 \%$ MFCP and $16.67 \%$ PC cases, whereas, they obstructed the lesion area in $8.33 \% \mathrm{MFCP}$ and $70 \% \mathrm{PC}$ cases. These data were consistent with previous literature (3), and are characteristic of PC.

CT multi-phase scan was the best imaging method for the early diagnosis of PC. It identified hemodynamic changes in $\mathrm{PC}$, and the degree of early enhancement was proportional to the vascular density and diffusion of the contrast agent into the extravascular space. Lack of blood supply in the PC tissue, especially in the arterial phase of the enhancement scan was characteristic of lesions in PC. Moreover, necrosis of a large number of normal pancreatic cells resulted in delayed clearance of the contrast agent. Thus, the performance of the dynamic enhancement (lower enhancement in artery phase and delayed enhancement in delayed phase) was slow lift platform type. The strengthening degree was higher in the arterial and portal venous phases of MFCP than in the PC group. The optimal cutoff value for differentiating MFCP from PC was $21.85 \mathrm{Hu}$ for arterial phase, and $37.70 \mathrm{Hu}$ for portal phase. Previous study also showed that the decrease of blood flow and blood volume was more pronounced in PC than in MFCP (11), which resulted in delayed peak extension in PC than in MFCP (3).

We also investigated if the peripancreatic vessels were involved in MFCP and PC. We observed narrowing fat gap between the lesions and the peripancreatic vessels, which were wrapped by the abnormal mass, especially in the PC group, and displaced with a shape-rule margin in MFCP. Furthermore, in most MFCP cases, we observed expansion of the superior mesenteric vein, probably because of blood flow changes as a result of pancreatitis.

In both MFCP and PC, most lymph nodes were enlarged around the pancreatic head, especially in PC patients. The PC-related lymph nodes were significantly enlarged and showed greater enhancement with fusion, heterogeneous enhancement and uneven thickness of the ring wall. However, these parameters were not statistically significant when compared with MFCP. In MFCP patients, the pancreatic nodes showed slight enlargement with edge blur and mild homogeneous enhancement. Moreover, these findings were difficult to distinguish from lymph node tuberculosis and needed to be combined with other clinical features for diagnosis.

Despite some similarities, DWI is critical in the early and differential diagnosis of MFCP and PC because it quantitatively evaluates the free movement of water molecules within the pancreatic tissue $(12,16,18,27)$. Most PC cases showed a high signal and lower ADC value on DWI than in MFCP because of low cell density in the extracellular space. Moreover, MFCP showed differences in the diffusion of water molecules in the lesion area because of differences in exudation and varying degrees of hyperplasia due to prolonged chronic inflammation. Our study shows that restricted diffusion of water molecules was more common and that the signal was significantly uneven in the PC group than in MFCP. These data are consistent with previous reports $(12,16,27)$. However, 
there is a considerable overlap in the DWI signals for both PC and MFCP. Hence, differential diagnosis requires comprehensive analysis of multi-modality imaging data. We selected a high b value of $800 \mathrm{~s} / \mathrm{mm}^{2}$ because studies have indicated that high $b$ values result in acquiring the true ADC value of the tissue and increase the specificity of the diagnosis $(12,16,18)$.

Most PCs show high uptake of ${ }^{18} \mathrm{~F}-\mathrm{FDG}$ by localized lesions in the early and delayed phases $(5,19)$. Our study showed that SUV values were lower for the MFCP than the PC group in both the early $(2.51 \pm 0.42$ vs. $7.13 \pm 3.04)$ and delayed $(2.10 \pm 0.31$ vs. $9.20 \pm 3.56)$ phases. The optimal cutoff SUV values were 4.85 (sensitivity of $90 \%$, and specificity of $92 \%$ ), and 4.90 (sensitivity of $97 \%$ and specificity of $96 \%$ ) for early and delayed phases to differentiate MFCP and PC, respectively. The SUV values of PC group increased with the delay time, whereas there was minimal change or a decrease in the MFCP group, consistent with previous reports $(5,13,19)$. However, macrophages, neutrophils and other inflammatory cells, which express the glucose transporter and glycolytic enzymes highly, also lead to accumulation of ${ }^{18} \mathrm{~F}-\mathrm{FDG}$ locally. This makes it difficult to distinguish $\mathrm{PC}$ from pancreatitis, tuberculosis and autoimmune diseases $(5,13)$. A case of MFCP, which was secondary to autoimmune pancreatitis, was confirmed by surgery and pathology in our study. The high-uptake of ${ }^{18}$ F-FDG by the multi-site uneven lesions, combined with other data from conventional CT scan and enhanced imaging showed clear signs of pancreatitis. Thus, comprehensive analysis of multiple multi-modality imaging data is necessary in most cases for accurate diagnosis. Previous studies showed high ${ }^{18} \mathrm{~F}$-FDG uptake in autoimmune pancreatitis (100\%). In most pancreatitis lesions or benign tumors, the high uptake of ${ }^{18}$ F-FDG was not smooth, non-nodular, and unevenly distributed, whereas, in most malignant lesions the uptake was smooth, localized, nodular and uniformly distributed $(5,13)$. Size of the pancreatic lesions also influenced the accuracy of ${ }^{18}$ F-FDG PET diagnosis because lesions with few tumor cells or low expression of the glucose transporter combined with limited spatial resolution and density contrast were easily missed in a PET/CT scan (5). In contrast, smaller malignant tumors with high glucose metabolism, and mucus or serous cystadenocarcinoma exhibited low ${ }^{18} \mathrm{~F}$-FDG uptake $(5,13)$. In our study, many PC cases showed the above-described negative findings, but had characteristic imaging findings on dynamic contrast-enhanced CT and delayed PET/CT scans. Early or malignant PC lesions that are smaller show negative PET/CT. Therefore, delayed imaging combined with enhanced CT or MRI is essential for accurate and differential diagnosis. Accuracy of PC diagnosis with ${ }^{18} \mathrm{~F}-\mathrm{FDGPET} / \mathrm{CT}$, MDCT and MRI was 89,76 and $70 \%$, respectively $(3,5)$. PC was more accurately diagnosed by PET/CT, which also showed higher sensitivity for distant metastasis than MDCT and MRI. Moreover, SUV levels were influenced by factors such as weight of the individual, fat, blood glucose level, and selection of image reconstruction method (13).

This study is mainly discussing the multimodal imaging features and its differential diagnosis efficiency on distinguishing PC from MFCP of the pancreatic head. The comparison analysis of diagnostic efficacy among different single method of imaging examination will be presented in next research. It is also well know that any single imaging examination has some overlapping and limitations. Therefore, tumor marker results were not included in the analysis which will be taken into account in the next study, but our results indicate that the CA19-9 value were increased in 21 cases with PC (average $340.34 \pm 140.65 \mathrm{U} / \mathrm{dl}$ ) and in 13 cases with MFCP (average 109.05 $\pm 37.87 \mathrm{U} / \mathrm{dl}$ ).

In conclusion, we demonstrate that integration of dynamic contrast-enhanced CT scan MRI and ${ }^{18} \mathrm{~F}-\mathrm{FDG}-\mathrm{PET} / \mathrm{CT}$ imaging methods is necessary for differential diagnosis of MFCP from PC. This study was a retrospective study with a limited sample size and therefore needs to be confirmed by large scale study. In addition, the clinical stage (TNM staging) of PC was not analyzed which will be covered in the future study along with possible molecular mechanism (28-30).

\section{Acknowledgements}

Not applicable.

\section{Funding}

The present study was funded by The Department of Science and Technology in Guiyang (grant no. GY2015-32).

\section{Availability of data and materials}

The datasets used and/or analyzed during the current study are available from the corresponding author on reasonable request.

\section{Authors' contributions}

RZ designed the research and wrote the manuscript. MD performed the statistical analysis. RZ, JJ, QJ, LJ, CJ, LQ and WC collected, analyzed and interpreted all of the data and results. All authors read and approved the final manuscript.

\section{Ethics approval and consent to participate and publication}

The present study was approved by the Ethics Committee of the Affiliated Hospital of Guizhou Medical University (Guizhou, China) and was performed according to the Declaration of Helsinki guidelines. Written informed consent was obtained from all participants for inclusion in the study.

\section{Consent for publication}

Written informed consent was obtained from all participants for publishing their images in this manuscript.

\section{Competing interests}

The authors declare that they have no competing interests.

\section{References}

1. Dutta AK and Chacko A: Head mass in chronic pancreatitis: Inflammatory or malignant. World J Gastrointest Endosc 7: 258-264, 2015.

2. Merdrignac A, Sulpice L, Rayar M, Rohou T, Quehen E, Zamreek A, Boudjema K and Meunier B: Pancreatic head cancer in patients with chronic pancreatitis. Hepatobiliary Pancreat Dis Int 13: 192-197, 2014. 
3. Yao L, Jian Z and Jing ZC: Differential diagnosis of imaging between the mass-forming chronic pancreatitis and pancreatic carcinoma. Chin J Pancreatol 15: 59-63, 2015.

4. Braganza JM, Lee SH, Mccloy RF and Mcmahon M J: Chronic pancreatitis. Lancet 377: 1184-1197, 2011.

5. Kato K, Nihashi T, Ikeda M, Abe S, Iwano S, Itoh S, Shimamoto K and Naganawa S: Limited efficacy of (18)F-FDG PET/CT for differentiation between metastasis-free pancreatic cancer and mass-forming pancreatitis. Clin Nucl Med 38: 417-421, 2013.

6. Perumal S, Palaniappan R, Pillai SA, Velayutham V and Sathyanesan J: Predictors of malignancy in chronic calcific pancreatitis with head mass. World J Gastrointest Surg 5: 97-103, 2013.

7. Raimondi S,Lowenfels AB, Morselli-Labate AM, Maisonneuve P and Pezzilli R: Pancreatic cancer in chronic pancreatitis; aetiology, incidence, and early detection. Best Pract Res Clin Gastroenterol 24: 349-358, 2010

8. Min HS, Qiang LZ, Xiao Y, Chan Y, Wei ZH, Wen WD and Jun S: Clinical efficacy of pancreaticoduodenectomy and duodenum-preserving pancreatic head resection for the treatment of chronic pancreatitis with mass in the head of the pancreas. Chin J Dig Surg 14: 653-658, 2015.

9. Xue SA and Gong ZC, : Progress in diagnosis and surgical treatment for pancreatic head mass due to chronic pancreatitis. Chin J Gen Surg 25: 434-438, 2016.

10. Yi L, Ming LY, NaL, NaLX and Lin DB: Application of ${ }^{18} \mathrm{~F}$ FDG $\mathrm{PET} / \mathrm{CT}$ in the diagnosis and differential diagnosis of benign and malignant pancreatic lesions. J China Med Univ 43: 547-552, 2014

11. Lu N, Feng XY, Hao SJ, Liang ZH, Jin C, Qiang JW and Guo QY: 64-slice CT perfusion imaging of pancreatic adenocarcinoma and mass-forming chronic pancreatitis. Acad Radiol 18: 81-88, 2011.

12. Dale BM, Braithwaite AC, Boll DT and Merkle EM: Field strength and diffusion encoding technique affect the apparent diffusion coefficient measurements in diffusion-weighted imaging of the abdomen. Invest Radiol 45: 104-108, 2010.

13. Diederichs CG, Staib L, Vogel J, Glasbrenner B, Glatting G, Brambs HJ, Beger HG and Reske SN: Values and limitations of ${ }^{18} \mathrm{~F}$-fluorodeoxyglucose-positron-emission tomography with preoperative evaluation of patients with pancreatic masses. Pancreas 20: 109-116, 2000.

14. Ye S, Wang WL and Zhao K: F-18 FDG hypermetabolism in mass-forming focal pancreatitis and old hepatic schistosomiasis with granulomatous inflammation misdiagnosed by PET/CT imaging. Int J Clin Exp Pathol 7: 6339-6344, 2014.

15. Ying JS, Yun LX and Ju KH: Analysis of misdiagnosis of pancreatic cancer with ultrasound and its strategy. J Hepatopancreatobiliary Surg 25: 427-429, 2013

16. Ke NX, Yu YH, Quan XY, Feng S, Chuan ZL, Xuan WW and FengYH: Evaluation of diffusion-weighted imaging in distinguishing pancreatic carcinoma from mass-forming chronic pancreatitis: A meta analysis. J Clin Radiol 34: 70-74, 2015 .
17. $\mathrm{Li} \mathrm{H}$ and Yi XT: Progress in the value of CT, MRI and PET-CT in the diagnosis and staging of pancreatic cancer. J Med Postgra 27: 777-780, 2014

18. Choi SY, Kim SH, Kang TW, Song KD, Park HJ and Choi YH: Differentiating mass-forming autoimmune pancreatitis from pancreatic ductal adenocarcinoma on the basis of contrast-enhanced MRI and DWI findings. AJR Am J Roentgenol 206: 291-300, 2016.

19. Gu X and Liu R: Application of ${ }^{18} \mathrm{~F}-\mathrm{FDG}$ PET/CT combined with carbohydrate antigen 19-9 for differentiating pancreatic carcinoma from chronic mass-forming pancreatitis in Chinese elderly. Clin Interv Aging 11: 1365-1370, 2016.

20. Kim M, Jang KM, Kim JH, Jeong WK, Kim SH, Kang TW, Kim YK, Cha DI and Kim K: Differentiation of mass-forming focal pancreatitis from pancreatic ductal adenocarcinoma: Value of characterizing dynamic enhancement patterns on contrast-enhanced MR images by adding signal intensity color mapping. Eur Radiol 27: 1722-1732, 2017.

21. Falconi M, Bassi C, Casetti L, Mantovani W, Mascetta G, Sartiru N, Frulloni L and Pederzoli P: Long-term results of Frey's procedure for chronic pancreatitis: A longitudinal prospective study on 40 patients. J Gastrointest Surg 10: 504-510, 2006.

22. NeffCC, Simeone JF, Wittenberg J, Mueller PR and Ferrucci JT Jr: Inflammatory pancreatic masses. Problems in differentiating focal pancreatitis from carcinoma. Radiology 150: 35-38, 1984.

23. Dian ZE, Wu ZD and De LS: Clinical analysis of 39 cases of chronic pancreatitis with mass. Chin J Dig 29: 161-163, 2009.

24. Martinez E, Silvy F, Lombardo D and Mas E: Single nucleotide polymorphisms and risk factors predictive of pancreatic adenocarcinoma. Can Cell Microenviron 3: e1231, 2016.

25. Westphale CB, Renz BW, Reiche M, Rustg AK and Wan TC: Cellular plasticity and heterogeneity in pancreatic regeneration and malignancy. Can Cell Microenviron 3: e1472, 2016.

26. Pu N, Zhao G, Lv Y and Wu W: The advancement of tumor microenvironment in pancreatic carcinoma. Can Cell Microenviron 3: e1248, 2016.

27. Frulloni L, Amodio A, Katsotourchi AM and Vantini I: A practical approach to the diagnosis of autoimmune pancreatitis. World J Gastroenterol 17: 2076-2079, 2011.

28. Krueger J, Jules F, Reider S and Rudd C: CD28 family of receptors inter-connect in the regulation of T-cells. Recept Clin Invest 4: e1581, 2017.

29. Adriani G, Bai J, Wong SC, Kamm RD and THIERY JP: M2a macrophages induce contact-dependent dispersion of carcinoma cell aggregates. Macrophage 3: e1222, 2016.

30. Runa F, Adamian Y and Kelber JA: Ascending the PEAK1 toward targeting TGF $\beta$ during cancer progression: Recent advances and future perspectives. Can Cell Microenviron 3: e1162, 2016.

This work is licensed under a Creative Commons Attribution-NonCommercial-NoDerivatives 4.0 International (CC BY-NC-ND 4.0) License. 